\title{
Hyperlipasemia in Pediatric Inflammatory Bowel Diseases
}

\author{
Faith D. Ihekweazu, M.D., \\ Department of Pediatric Gastroentoerology, Baylor College of Medicine, Texas Children's \\ Hospital, Houston, Texas \\ Dorottya Nagy-Szakal, M.D., \\ Department of Pediatric Gastroentoerology, Baylor College of Medicine, Texas Children's \\ Hospital, Houston, Texas \\ Douglas S. Fishman, M.D., and \\ Department of Pediatric Gastroentoerology, Baylor College of Medicine, Texas Children's \\ Hospital, Houston, Texas \\ Richard Kellermayer, M.D., Ph.D. \\ Department of Pediatric Gastroentoerology, Baylor College of Medicine, Texas Children's \\ Hospital, Houston, Texas
}

\section{To the Editor}

Acute pancreatitis is a known, albeit rare, complication of inflammatory bowel diseases (IBDs) $)^{1}$. In pediatric patients, acute pancreatitis is diagnosed based on the presence of two of the following three features: 1) abdominal pain characteristic of acute pancreatitis, 2) serum amylase or lipase $\geq 3$ times the upper limit of normal, and 3) characteristic findings of acute pancreatitis on imaging 2,3 . There is a subset of IBD patients (up to 20\%) reported to have hyperamylasemia and/or hyperlipasemia without the diagnosis of acute pancreatitis ${ }^{4}$. Therefore, this diagnosis can be difficult to make in the setting of IBD, as many patients will present with the complaint of abdominal pain secondary to their underlying disease, but with simultaneous elevation in pancreatic enzymes. We hypothesized that hyperlipasemia in the setting of pediatric IBD is rarely associated with radiographically proven pancreatitis, but is instead a unique feature of IBD activity itself.

We conducted a retrospective chart review of 275 pediatric IBD patients presenting to Texas Children's Hospital between January 2010 and December 2012. We specifically noted the type of IBD (crohn's disease [CD], ulcerative colitis [UC] or indeterminate colitis [IC]), laboratory examination for lipase and how often this was noted to be elevated, presence of thiopurine, 5-ASA and/or corticosteroid medications at the time of hyperlipasemia,

Correspondence: Faith Ihekweazu, MD, Section of Pediatric Gastroenterology, Hepatology \& Nutrition, Baylor College of Medicine, 6621 Fannin St., CC1010.00, Houston, TX 77030-2399, Voice: 281-797-5695, Fax: 832-825-3633, faith.ihekweazu@ bcm.edu.

Author Contributions: Faith Ihekweazu performed the acquisition of data, analysis and interpretation of data and drafting and revising of the manuscript. Dorottya Nagy-Szakal performed drafting and revising of the manuscript. Douglas Fishman revised the manuscript and provided significant intellectual contribution. Richard Kellermayer performed the conceptual design, data analysis and drafting and revising of the manuscript. All authors read and approved the final manuscript.

Conflicts of Interest: The authors have no conflict of interest to report. 
screening for IgG4 subclass, as well as radiographic imaging to support the diagnosis of pancreatitis. In those patients with hyperlipasemia, we correlated clinical findings at the time, and noted whether the diagnosis of acute pancreatitis was made.

Serum lipase was checked in 114 patients (41.5\%), and was found to be elevated in $19.3 \%$ ( 22 of the 114). However, only 9 patients (7.9\% of those checked; 5 CD, 4 UC) had a lipase that was $\geq 3$ times the upper limit of normal (ULN) (Table 1). All 9 patients had imaging of the pancreas during an episode of hyperlipasemia (including ultrasound, magnetic resonance enterography [MRE], magnetic resonance cholangiopancreatography [MRCP] and/or computed tomography [CT] scan). Importantly, none had evidence of pancreatitis on imaging. Overall $36.3 \%$ of the $>3$ fold ULN hyperlipasemia episodes ( 4 of 11 episodes) were attributed to drug-induced pancreatitis, $27.3 \%$ ( 3 of 11 ) were secondary to IBD flare, $27.3 \%$ ( 3 of 11 ) were noted during acute infections, and only 9\% (1 of 11 ) were diagnosed as idiopathic acute pancreatitis.

It is worth noting that normal pancreatic imaging was found in all of the patients who met the clinical criteria for pancreatitis (with abdominal pain and lipase $\geq 3$ times the upper limit of normal). In a pediatric cohort of non-IBD patients with acute pancreatitis, $70.6 \%$ (24 out of 34) had abnormal US findings ${ }^{5}$. Cross-sectional imaging (CT and MRI) are more sensitive techniques for diagnosing pancreatitis. ${ }^{6}$ Therefore, the consistent lack of pancreatic abnormalities by imaging in our cohort further brings into question the diagnosis of pancreatitis in the IBD patients studied, although meeting other clinical diagnostic criteria (i.e. abdominal pain, and lipase $>3 \times$ ULN).

Other studies have demonstrated hyperlipasemia in IBD patients. For example, a crosssectional Finnish study looked at the prevalence of elevated pancreatic enzymes in IBD. Seven percent (7\%) of the study population was noted to have hyperlipasemia, with $11 \%$ demonstrating hyperamylasemia ${ }^{4}$. Another prospective study of 136 IBD patients found $14 \%$ to have asymptomatic elevations in amylase and lipase, with no association between lipase elevation and IBD activity index or C-Reactive Protein (a marker of inflammation) ${ }^{7}$. Proposed explanations for this asymptomatic hyperlipasemia included latent extra-intestinal involvement of the pancreas, extra-pancreatic release of lipase from the inflamed bowel, and intestinal reabsorption of released lipase in the inflamed bowel ${ }^{7}$.

Autoimmune pancreatitis (AIP) can also complicate IBD, with AIP conferring a 12-15 fold increased risk for IBD when compared to the general population. ${ }^{8}$ It is possible that in a subgroup of patients with increased IgG4 in colonic mucosal specimens, the colitis may be an extrapancreatic manifestation of AIP mimicking $\mathrm{IBD}^{8}$. Notably, in our patient population IgG4 was only examined in 1 patient and was normal, making AIP an unlikely explanation for hyperlipasemia in that patient.

Another possible explanation of hyperlipasemia is macrolipasemia, a complex of lipase with other proteins, usually immunoglobulins. ${ }^{9}$ None of the patients in our cohort were studied for macrolipasemia, therefore it is unclear if this etiology is contributing to hyperlipasemia in this setting. 
In our population of pediatric IBD patients, we have shown that hyperlipasemia is a relatively common finding ( $20 \%$ of patients). We have also observed the difficulties of accurately diagnosing acute pancreatitis in the setting of pediatric IBD, given the overlap of symptoms in both conditions. Based on our observations, we conclude that hyperlipasemia may most often be a nonspecific complication of pediatric IBD. Therefore, we recommend careful consideration when diagnosing pancreatitis in pediatric IBD patients. MRI as the most sensitive diagnostic modality, considerations for autoimmune pancreatitis and macrolipasemia may aid the final conclusions and the selection of therapeutic interventions.

\section{Acknowledgments}

Funding: This work was supported by the National Institutes of Health [grant number T32 DK007664-24S1] to FDI; and the Gutsy Kids Fund supported by the Karen and Brock Wagner family; and the Houston Men of Distinction to RK. Funding sources had no role in the study design, in the collection, analysis and interpretation of data; in the writing of the manuscript; or in the decision to submit the manuscript for publication.

Acknowledgement of Contributors: We would like to acknowledge Dr. Seema Mehta Walsh's leadership in the Improve Care Now Registry at Texas Children's Hospital. We would also like to express our gratitude toward Yafit Horowitz for encouraging our group to perform this analysis.

\section{References}

1. Bermejo F, Lopez-Sanroman A, Taxonera C, et al. Acute pancreatitis in inflammatory bowel disease, with special reference to azathioprine-induced pancreatitis. Aliment Pharmacol Ther. 2008; 28:623-8. [PubMed: 18513380]

2. Banks PA, Freeman ML. Practice Parameters Committee of the American College of G. Practice guidelines in acute pancreatitis. Am J Gastroenterol. 2006; 101:2379-400. [PubMed: 17032204]

3. Morinville VD, Husain SZ, Bai H, et al. Definitions of pediatric pancreatitis and survey of present clinical practices. J Pediatr Gastroenterol Nutr. 2012; 55:261-5. [PubMed: 22357117]

4. Heikius B, Niemela S, Lehtola J, et al. Elevated pancreatic enzymes in inflammatory bowel disease are associated with extensive disease. Am J Gastroenterol. 1999; 94:1062-9. [PubMed: 10201484]

5. Haddock G, Coupar G, Youngson GG, et al. Acute pancreatitis in children: a 15-year review. J Pediatr Surg. 1994; 29:719-22.

6. O'Neill E, Hammond N, Miller FH. MR imaging of the pancreas. Radiol Clin North Am. 2014; 52:757-77. [PubMed: 24889170]

7. Bokemeyer B. Asymptomatic elevation of serum lipase and amylase in conjunction with Crohn's disease and ulcerative colitis. Z Gastroenterol. 2002; 40:5-10.

8. Ravi K, Chari ST, Vege SS, et al. Inflammatory bowel disease in the setting of autoimmune pancreatitis. Inflamm Bowel Dis. 2009; 15:1326-30. [PubMed: 19235915]

9. Keating JP, Lowe ME. Persistent hyperlipasemia caused by macrolipase in an adolescent. J Pediatr. 2002; 141:129-31. [PubMed: 12091864] 


\section{Table 1}

Frequency of elevated serum lipase in the total study population and in different categories of IBD. CD = Crohn's Disease; UC = Ulcerative Colitis; IC = Indeterminate Colitis

\begin{tabular}{|c|c|c|c|c|}
\hline & All Patients & CD & UC & IC \\
\hline No. of Patients (\%) & $275(100)$ & $159(57.8)$ & $97(35.2)$ & $19(6.9)$ \\
\hline Lipase evaluated (\%) & $114(41.5)$ & $68(42.8)$ & $40(41.2)$ & $6(31.6)$ \\
\hline Elevated lipase (\%) & $22(19.3)$ & $12(17.6)$ & $10(25)$ & 0 \\
\hline Lipase 23x ULN (\%) & $9(7.9)$ & $5(7.4)$ & $4(10)$ & 0 \\
\hline
\end{tabular}

